\title{
Design and application of enhanced communication protocols for wireless sensor networks operating in environmental monitoring
}

\section{F. Chiti, M. Ciabatti, G. Collodi, D. Di Palma and Romano Fantacci*}

Dipartimento di Elettronica e Telecomunicazioni, Universitá degli Studi di Firenze, Italy

E-mail: chiti@lenst.det.unifi.it

E-mail: michele.ciabatti@unifi.it

E-mail: collodi@ing.unifi.it

E-mail: davide.dipalma@unifi.it

E-mail: romano.fantacci@unifi.it

*Corresponding author

\author{
A. Manes \\ Dipartimento di Energetica, \\ Universitá degli Studi di Firenze, Italy \\ E-mail: antonio.manes@unifi.it
}

\begin{abstract}
The effective application of Wireless Sensor Networks (WSNs) to wide area environmental monitoring currently represents a challenge for this technology. Since devices are usually energy constrained, smart power saving procedures are needed to increase the network lifetime. A common approach is to switch off the node's radio; this definitely requires the adoption of synchronisation procedures that properly schedule packets transmission to avoid both overhearing effects and collisions. To this end, a novel class of MAC layer protocols, named STAR MAC, is presented. This is further improved by introducing STAR+ scheme and properly integrating within a routing solution, according to the cross-layer design. It allows the optimisation of energetic consumption, or equivalently, network life-time, together with minimising the signalling overhead. The proposed solution is then applied to a realistic user-defined scenario, highlighting remarkable advantages both in terms of robustness, self reconfigurability, cost, complexity and QoS, thus validating the proposed solution.
\end{abstract}

Keywords: wireless sensor networks; WSNs; MAC protocols; energy efficiency; synchronisation.

Reference to this paper should be made as follows: Chiti, F., Ciabatti, M., Collodi, G., Di Palma, D., Fantacci, R. and Manes, A. (2007) 'Design and application of enhanced communication protocols for wireless sensor networks operating in environmental monitoring', Int. J. Sensor Networks, Vol. 2, Nos. 5/6, pp.299-310.

Biographical notes: Francesco Chiti (M'00) received the Telecommunications Engineering and the $\mathrm{PhD}$ in Informatics and Telecommunications Engineering degrees from the University of Florence in 2000 and 2004. His current research topics are devoted to MAC, LLC and network layers protocols for both public and private wireless communications systems and ad hoc and sensor networks as well. He took part in several European research projects as IP GoodFood, the NoEs Nexway, SatNex, Newcom and CRUISE, the GJU TWIST, the ETSI STF179 and the COST 289 action.

Michele Ciabatti received the Laurea degree in Telecommunication Engineering from the University of Florence Italy, in 2004, with a thesis on performance and error analysys of water steam reconstruction algorithms through active measures in LIMB mode. Since January 2005, he is a PhD student on Wireless Sensor Network Communication protocols.

Giovanni Collodi received the Laurea and the $\mathrm{PhD}$. degrees in Electronic Engineering from the University of Florence, Florence, Italy, in 1996 and 2000, respectively. Since 1996, he joined the Department of Electronics Engineering, University of Florence, where he became an Assistant Professor in 2004. During his academic career, he has been Lecturer in courses of Applied Electronics, and now he teaches Integrated Circuit for Wirelss Application. His research 
activities have covered the study of active and passive compact structures for MMICs, the design of multifunction MMICs for low-power wireless applications, CAD and numerical modelling. Currently, he is working on applied researches on wireless sensor network and their applications on environmental monitoring.

Davide Di Palma received a Laurea degree in Biomedical Electronic Engineering from the University of Florence, Florence, Italy in July 2004. In 2005, he joined the MicLab: currently, he is a $\mathrm{PhD}$ student and his main research activity is related to the study and the realisation of wireless sensors networks, using new protocols and different boards.

Romano Fantacci, received the Dr Ing degree in Electronics Engineering from the University of Florence, Florence, Italy in 1982 and a PhD in Telecommunication Engineering in 1987. In 1982, he joined in the Department of Electronics and Telecommunications in the University of Florence, first as Researcher, then as an Associate Professor, and currently as Full-time Professor. His current research interests are digital communications, computer communications, queuing theory, satellite communication systems, wireless broadband communication networks, ad hoc and sensor networks. He has been involved in several national, European and nationwide research projects. He is IEEE Fellow Member and takes part in several IEEE COMSOC Committees together with being Guest Editor of IEEE COMSOC journal special issues and Member of the Editorial Board of several international journals.

Antonio Manes after receiving his degree in Telecommunication Engineering from the University of Florence in 2002, he started a PhD course on Energetic and Industrial Technologies, completed in 2005 with a thesis on Wireless Sensor Networks for Industrial Monitoring and Control. Still working at the Department of Energetics, he is involved in EU founded research projects of the 6th FP; actually his research is oriented on development of low power wireless devices and protocols for industrial applications.

\section{Introduction}

In recent years, the availability of on-field monitoring became a key issue for the assessment of environmental processes and parameters. In this context, the application of Wireless Sensor Networks (WSNs) technology represents a significant advance over traditional invasive methods of monitoring as pioneering envisioned in Akyildiz et al. (2002) and applied to several fields in Hać (2003). As a matter of fact, instrumenting natural spaces with networked microsensors might enable long-term data collection with enhanced accurateness. Further, the communication capabilities allow nodes to cooperate in performing more complex tasks, like statistical sampling or data aggregation, not feasible with a point-to-point telemetry. Finally, the computing and networking capabilities permits both the reprogramming or even the self-reconfiguring of the whole system.

The increasing need for products controlling at different critical steps of the agrofood chain open a novel application field for WSN. Among the related initiatives founded by public institutions, the GoodFood Integrated Project (http://www.goodfood-project.org), presented within the Information Society Technology (IST) thematic area of European Community Sixth Frame Project (EU VI FP), aims at developing the new generation of analytical methods based on Micro and Nanotechnology (M\&NT) solutions for the safety and quality assurance along the food chain in the agrofood industry. GoodFood approach will comply, through the development of innovative M\&NT solutions, with the needs of ubiquity, low cost and low power, fast response, simple use and fully interconnection to the decisional bodies. The project's WorkPackage 7, aimed at implementing intelligent and innovative communication solutions for food safety and quality traceability, is developing a Pilot Site, oriented to the wine production phase monitoring; this Pilot Site is being deployed at the Montepaldi Farm (in the Chianti region, Italy) and is expected to be fully operative by the end of 2005 .

The requirements that WSN adoption is expected to satisfy in performing an effective agrofood monitoring are mainly concerned with the operating period. In particular, a large number of battery operated nodes, deployed in outdoor environments, is expected to operate at least for a farming season (usually 3-6 months), without maintenance. Besides, since node are usually unattended, they might cope with node fails or network congestion by means of adapting or even self-reconfiguring their functionalities and protocols. Finally, correct data delivering to remote user via gateway is to be guaranteed to allow a proper monitoring.

The aforementioned requirements compels the WSN communications protocols to be carefully designed and optimised. In this paper, a cross-layer solution is presented, joining the Medium Access Control (MAC) and network layers operations and applying it to a practical case study, allowing to develop self configuring, long life-time and effective network infrastructure and to satisfy user-oriented specifications. The MAC layer protocol features are described in Section 2 together with the performance analysis as to the network synchronisation capabilities. The protocol has been analytically characterised and generalised to the case of completely independent nodes in terms of frame period and duty cycle values. Further, it is 
modified in order to cope with densely deployed networks, giving rise to the Enhanced Synchronous Transmission Asynchronous Reception (STAR+) MAC and the provided gain is investigated within the Appendix. In Section 2.4 the cross-layer routing protocol is highlighted and the overall communications performance is presented for the practical test case offered by the GoodFood project. Finally, some conclusions are drawn explaining the future directions of the present research activity.

\section{MAC layer protocol}

\subsection{System requirements}

The most relevant system requirements leading the design of an efficient MAC protocol for an environmental monitoring WSN, as to GoodFood project, are mainly concerned with the power consumption issues and the capability of quickly setup an end-to-end communication infrastructure that supports both synchronous and asynchronous queries. In particular, the most relevant challenge is to make a system able to run unattended for a long period, as nodes are expected to be deployed in zones hardly manageable for maintenance. This calls for an optimal energy management, since it is anyhow a limited resource and the failure of a nodes may compromise WSN connectivity, as the network gets partitioned. Therefore, the MAC layer is to be optimised ensuring that the energy it spends is directly related to the amount of traffic that it handles and not to the overall working time.

Other important properties are scalability and adaptability of network topology, in terms of number of nodes and their density. As a matter of fact, some nodes may either be turned off or join the network afterward or finally, they could be moved to a different location. Thus, the MAC layer protocol might be able to face these events with an higher degree of responsiveness and reconfigurability.

Finally, several user-oriented attributes, including fairness, latency, throughput and bandwidth utilisation, need to be taken into account even if they could be considered secondary with respect to our application purposes, since, in contrast to typical WLAN protocols, MAC protocols designed for WSN usually trade-off performance for cost (Langendoen and Halkes, 2005).

Taking the IEEE 802.11 Distributed Coordination Function (DCF) (IEEE standard 802.11, 1999) as a starting point, several more energy efficient techniques have been proposed in literature to avoid excessive power wasting when a node are in the so-called idle mode, that is, a state in which it continuously sample the channels waiting for a message their circuitry. They are based on the receiver periodical turning on the radio to detect the presence of a packet preamble and if no signal is sensed the radio is turned-off again until the next sample. As a consequence, a low power state, in which the transceiver is disabled and only a low level clock is active, might be introduced, duty cycling the radio. Furthermore, the sender can simply wait until the moment the receiver is about to sample the channel and send a packet with an ordinary preamble, thus saving energy both at the sender and at the receiver. In particular, according to the WiseMAC protocol (El-Hoiydi et al., 2003) nodes maintain the schedule offsets of their neighbours through piggy backed information on the ACK of the underlying CSMA protocol. However, WiseMAC suffers the disadvantages of being subject to traffic fluctuations and moreover, to not efficiently managing message broadcasting due to the absence of synchronisation procedures.

Deriving from the classical contention-based scheme, several protocols (S-MAC (Ye et al., 2002), T-MAC (van Dam and Langendoen, 2003) and DMAC (Lu et al., 2004)) have been proposed to address the idle listening overhead by synchronising the nodes and implementing a duty cycle within each slot. At the beginning of a slot, all nodes wakeup and any node wishing to transmit a message must contend for the channel. This increases the probability of collision in comparison to the random organisation of the energy-efficient CSMA protocols. To mitigate the increased collision overheads S-MAC and T-MAC include an RTS/CTS handshake, while DMAC avoids this protocol overhead.

Finally, these approaches differ in their way of deciding when and how to switch back from active to sleep mode. In particular, in S-MAC protocol, neighbouring nodes form virtual clusters so as to set up a common sleep schedule. If two neighbouring nodes reside in two different virtual clusters, they wake up at the listen periods of both clusters. On the other hand, in T-MAC, the listen period ends when no activation event has occurred for a certain time threshold. Finally, DMAC scheme performs as an improved Slotted Aloha algorithm in which slots are assigned to the sets of nodes based on a data gathering tree.

Resorting to the above considerations, a class of MAC protocols, particularly suited for a flat network topology, as that considered in Goodfood project (http://www. goodfood-project.org), has been derived, taking the benefits of both WiseMAC and S-MAC schemes. In particular, it joins the power saving capability, due to the introduction of a duty cycle, together with the advantages provided by the offset scheduling, without an excessive signaling overhead. It is basically based on periodical rendez vous synchronisation of all possible couples of nodes by means of MAC layer messages to allow both communications and power saving. Further, to avoid excessive signalling overhead especially when the number of neighbours becomes larger protocol enhancement is proposed. More details about protocol issues, related TinyOS modules for the implementation and the provided advantages are given within the next section.

\subsection{Proposed scheme}

\subsubsection{Synchronous transmission asynchronous reception MAC}

An alternative approach to the problem of providing an energy efficient MAC solution is represented by the proposed scheme that is characterised as it follows. Following El-Hoiydi et al. (2003), each node might be either into an idle mode, in which it remains for a time interval $T_{1}$ (listening time) or in an energy saving sleeping state for a $T_{\mathrm{s}}$ (sleeping time). The transitions between states are synchronous with a period called frame equal to $T_{\mathrm{f}}=T_{1}+T_{\mathrm{s}}$ partitioned in two subintervals; as a 
Figure 1 STAR MAC characterisation for four different nodes

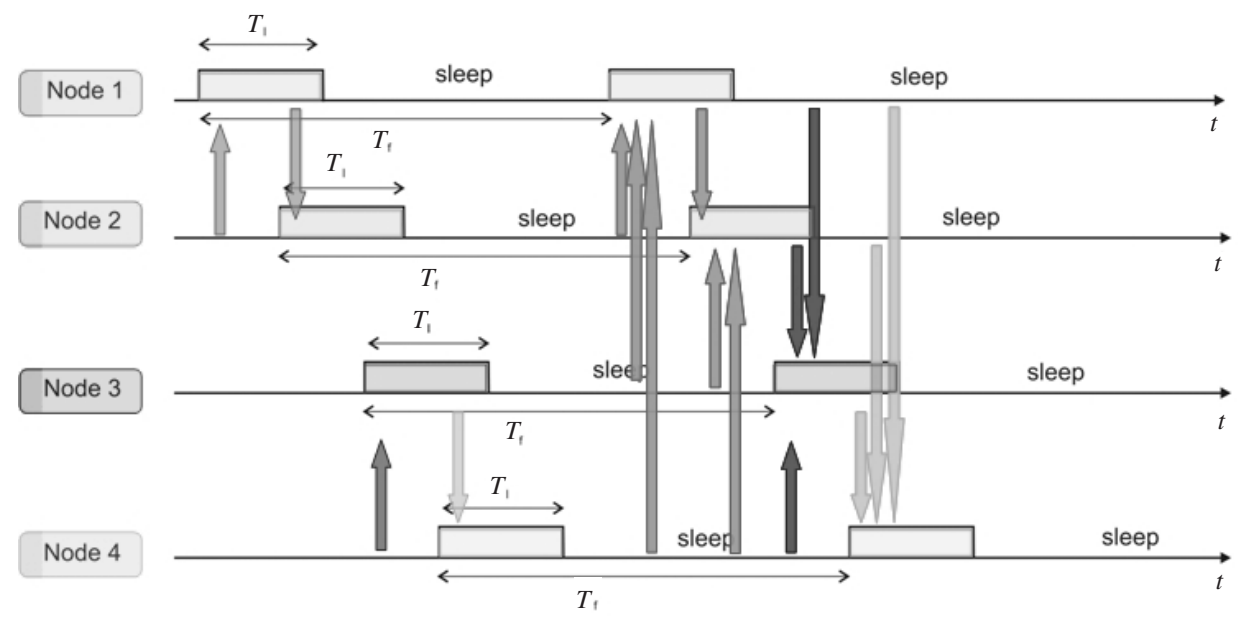

consequence, a duty cycle function can be also introduced:

$$
d=\frac{T_{1}}{T_{1}+T_{\mathrm{s}}}
$$

Though the protocol is able to manage a specific duty cycle for each node, it has been assumed to be the same for all the nodes as so it usually happens. To provide full communication capabilities to the network, all the nodes need to be weakly synchronised or equivalently, to be aware at least of the awakening time of all their neighbours. To this end, a node sends frame by frame one synchronisation message to each of its neighbour nodes known to be in the listening mode (synchronous transmission), as explained in Figure 1. Instead, during the set up phase in which each node is discovering the network topology, the control messages are asynchronously broadcasted. On the other hand, its neighbours periodically awake and enter the listening state independently (asynchronous reception), as it is again presented in Figure 1. The header of the synchronisation message contains the following fields: a node unique identifier, the message sequence number and the phase, that is, the time interval after which the sender enters a new frame period claiming to be in the listening status waiting for both the synchronisation and data messages from its neighbours. The phase $\phi$ is evaluated according to the Figure 2 as it follows:

$$
\phi_{1}=\tau+T_{\mathrm{S}}
$$

if the node is in the sleeping mode, where $\tau$ is the time remaining to the end of the actual listening period (or the next one if the node is in the sleeping state). Conversely, if the mote is in the listening status, $\phi$ is calculated as:

$$
\phi_{2}=\tau-T_{1}
$$

In order to fully characterise the performance of the Synchronous Transmission Asynchronous Reception (STAR) MAC approach, energetic consumption needs to be analytically described. According to our approach, it has been evaluated in $\mathrm{mAh}$ to be in compliance with the power battery supply. ${ }^{1}$
Figure 2 Phase evaluation for two different cases

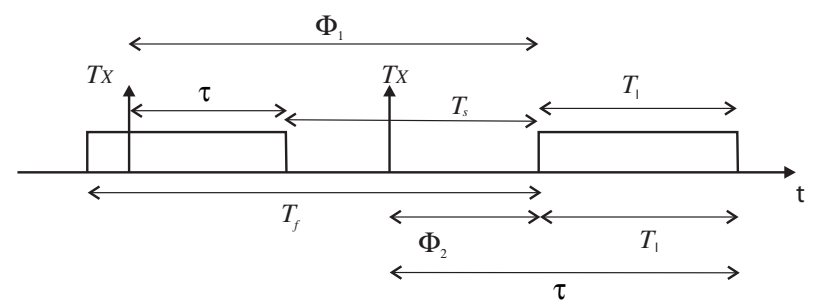

The energy spent for STAR MAC operation $(C)$ within one frame period can bed characterised as it follows:

$C=c_{\mathrm{Rx}} \mathrm{d} T_{\mathrm{f}}+c_{\text {sleep }}\left[T_{\mathrm{f}}(1-d)-N T_{\mathrm{pkt}}\right]+N C_{\mathrm{Tx}} \quad[\mathrm{mAh}]$

where $c_{\text {sleep }}$ and $c_{\mathrm{Rx}}$ represent the sleeping and the receiving costs [mA] and $C_{\mathrm{Tx}}$ is the single packet transmission costs [mAh], $T_{\mathrm{pkt}}$ is the synchronisation packet time length [s] and finally $N$ is the number of neighbours. In (4) only $T_{\mathrm{f}}$ and $d$ have been mentioned as they are the parameters that could be varied in practice. In effect, the previous equation might be generalised as the term $C_{\mathrm{Tx}}$ is different if a node attempts to transmit a packet while it is in the sleeping state. In this case, it first turns on the radio, then performs a channel sensing whose duration is related to the adopted MAC policy, transmits the packet and finally it turn off the radio. The most predominant factor is clearly the cost of the channel sensing that usually takes twice the packet duration. When the following inequality hold:

$$
N T_{\mathrm{pkt}} \ll T_{\mathrm{f}}
$$

then:

$$
C \cong c_{\mathrm{Rx}} d T_{\mathrm{f}}+c_{\text {sleep }} T_{\mathrm{f}}(1-d)+N C_{\mathrm{Tx}} \quad[\mathrm{mAh}]
$$

The protocol cost normalised to the synchronisation time is finally:

$$
\frac{C}{T_{\mathrm{f}}}=c_{\mathrm{Rx}} d+c_{\text {sleep }}(1-d)+\frac{N C_{\mathrm{Tx}}}{T_{\mathrm{f}}} \quad[\mathrm{mA}]
$$

As highlighted in Table 1, it usually happens that $c_{\mathrm{Tx}} \ll c_{\text {sleep }} \ll c_{\mathrm{Rx}}$, in where $c_{\mathrm{Tx}}=C_{\mathrm{Tx}} / T_{\mathrm{pkt}}$ and 
$T_{\mathrm{pkt}}$ is the packet transmission time [s], assumed equal to $100 \mathrm{~ms}$ as worst case. This means that the major contribution to the overall cost is represented by the listening period that the STAR MAC protocol tries to suitably minimise. Nevertheless, an advanced approach is presented below, aiming at minimising also the cost of packet transmission for densely deployed WSN or with a high traffic load factor.

Table 1 Power consumption values

\begin{tabular}{ll}
\hline Parameter & Value \\
\hline$c_{\mathrm{Rx}}$ & $\cong 10 \mathrm{~mA}$ \\
$c_{\text {sleep }}$ & $\cong 0.01 \mathrm{~mA}$ \\
$C_{\mathrm{Tx}}$ & $\cong 30 \mathrm{mAh}$ \\
$c_{\mathrm{Tx}}$ & $\cong 0.001 \mathrm{~mA}$ \\
\hline
\end{tabular}

\subsubsection{Enhanced STAR (STAR+) MAC}

This approach introduces an improvement in the STAR MAC as only one synchronisation packet is multicasted to all the neighbour nodes belonging to a subset, that is, such that they are jointly awake for a time interval greater than $T_{\mathrm{Rx}}$. This leads to an additional advantage, as the number of neighbours increases allowing better performance with respect to scalability and a power saving too. Besides, the synchronisation overhead is reduced with a consequent collisions lowering if no carrier sensing is performed. However, in the proposed approach, a Carrier Sensing Multiple Access (CSMA) scheme is adopted, allowing a benefit in terms of energy consumption and latency lowering. Under this hypothesis and moreover, supposing that the number of subsets is $K$, then the normalised cost might be expressed as:

$$
\frac{C}{T_{\mathrm{f}}}=c_{\mathrm{Rx}} d+c_{\text {sleep }}(1-d)+\frac{K C_{\mathrm{Tx}}}{T_{\mathrm{f}}} \quad[\mathrm{mA}]
$$

Since $K \leq N$, the normalised cost results to be remarkably lowered, especially if number of nodes and duty cycle get higher, even if the latter case is inherently power consuming.

\subsection{STAR MAC protocol performance analysis}

The evaluation of the proposed MAC protocol effectiveness might take into account two different aspects: first of all the energetic consumption or equivalently, the network life-time and also the capability of synchronise the nodes according to application requirements, that is in a weak sense.

In Figure 3 the normalised cost versus the number of neighbour nodes is shown for the S-MAC and STAR MAC schemes. It is worth noticing that the performance of the proposed protocol is better with respect to the existing approach for a number of neighbour nodes greater than 7 . For a number of neighbour nodes extremely high (not reported in Figure 3), the STAR+ protocol presents the advantage of a additional power consumption reduction, as the multicast effect becomes relevant. However, STAR+ is able to lower the collision probability by reducing the synchronisation messages.

Finally, in Figure 4 the normalised costs of S-MAC and STAR approaches are compared with respect to the duty cycle duration for a number of neighbour nodes equal to 8 . It is possible to notice that for $d<3.5 \%$ the proposed protocol provide a significant gain.

As far as the second aspect is concerned, it is worth noticing that a distributed network, in which nodes continuously switch on and off, involves the considerable task of synchronism maintaining among neighbouring nodes: small hardware tolerances that are negligible in an isolated device become relevant in long life networks, where motes have to preserve mutual communication. In particular, nodes might face timing misalignments (drift) arising from several reasons: different Finite State Machines (FSMs), technologies and implementations for each node or in addition not equal dynamic behaviours due to different sensing messages handling. As a result, within a short period, it is likely the lack of synchronisation for the nodes to occur.

Figure 3 Normalised cost versus number of neighbour nodes

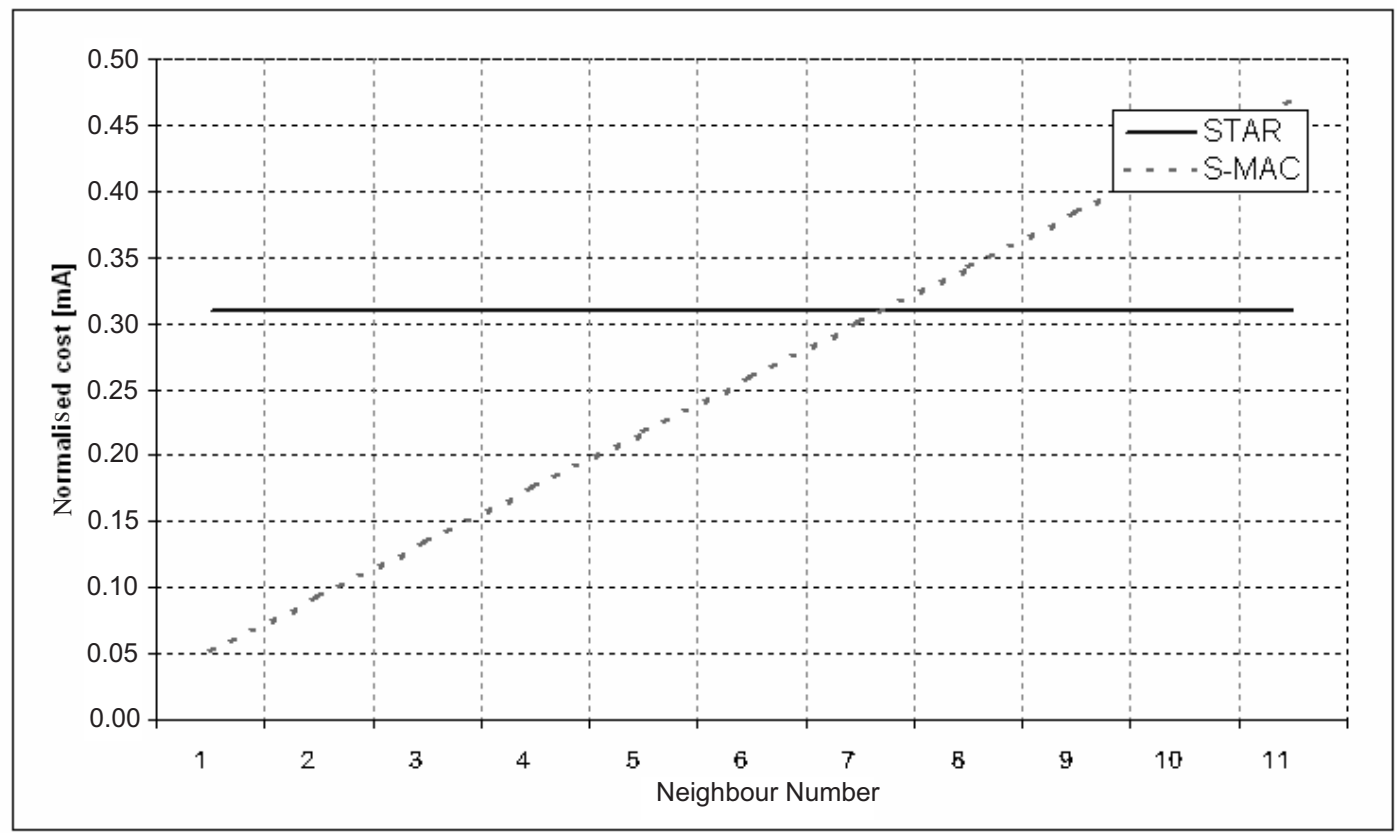


Figure 4 Normalised cost versus duty cycle duration

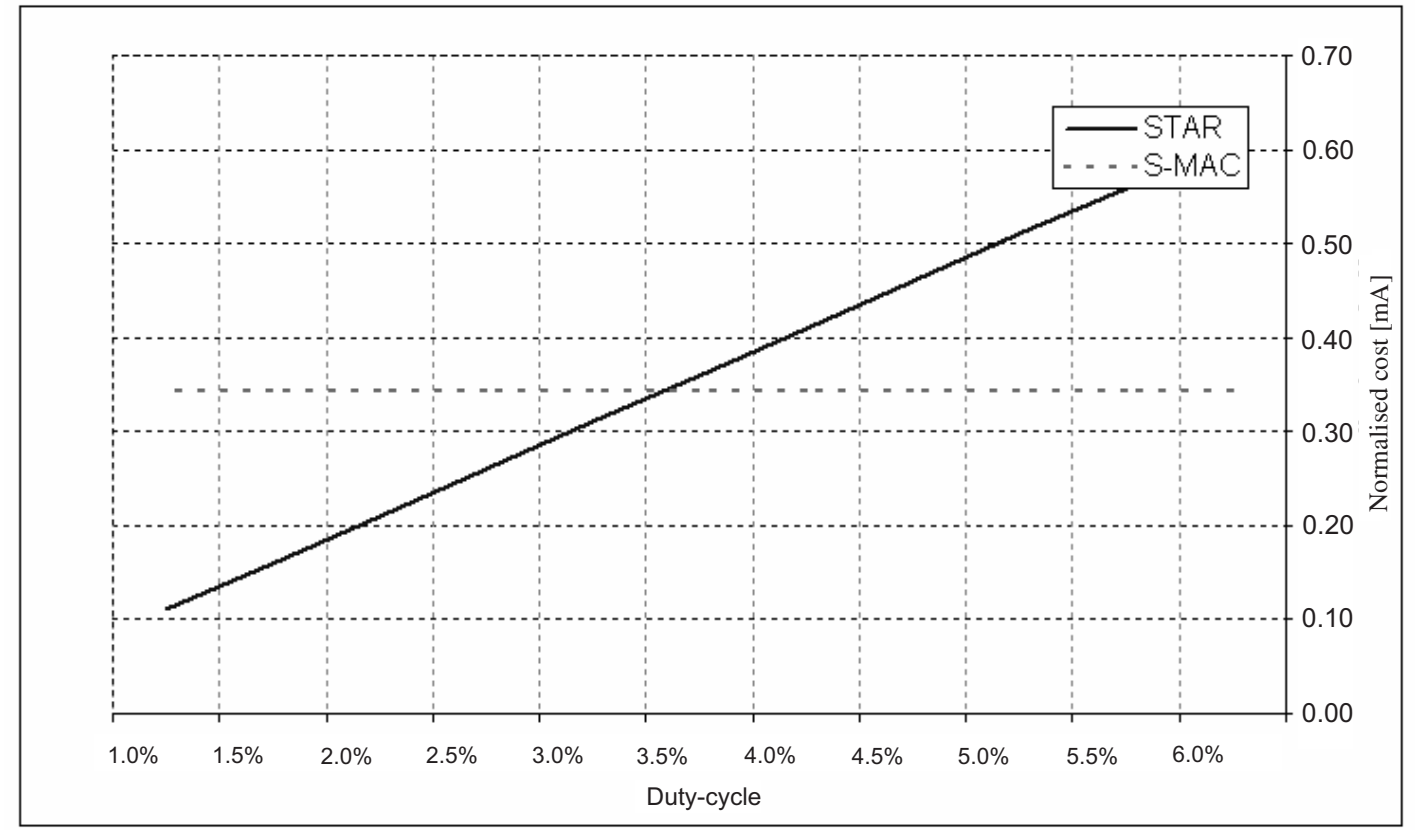

STAR MAC protocol effectively overcomes this problem, since it does not rely upon a strict distributed synchronisation: the nodes are not expected to behave exactly in the same way for what time issues are concerned, but the capabilities provided by the communication protocol permit to evaluate when and whom a message might be transmitted to. The dynamic phase estimation, performed every MAC period according to (2) or (3), allows each node to track and compensate the time drifts of its neighbours without additional complexity. Finally, it is worth noticing that the randomness of the awakening times of all the nodes, due to the deployment procedures, is able to reduce the collision probability over the shared medium.

To highlight the benefits yielded by the STAR MAC, two different test cases are presented. In the first case, two similar nodes with the same FSM and moreover with the same $T_{\mathrm{f}}$ and duty cycle equal to $60 \mathrm{sec}$ and $10 \%$, respectively, are investigated. In Figure 5 the time diagram concerning the values of the phase $\phi$ transmitted by each node is presented, pointing out the presence of a phase drift for each node due to a not ideal duty cycle. However, this is upper bounded by the communication protocol, since the neighbour node upon the reception of a synchronisation message is able to compensate for it by anticipating or delaying the sending of its next synchronisation message to that neighbour. Besides, this positive attitude is better highlighted in Figure 6 where the drift statistics for the nodes are compared. In particular, it is possible to show by means of numerical processing that these approximately random variables are both Gaussian with zero mean and the same variance, thus proving the goodness of our approach.

To fully characterise the system performance, a scenario comprising two nodes with different behaviours was also investigated. In particular, an ordinary node with $T_{\mathrm{f}}$ and duty cycle equal to $60 \mathrm{sec}$ and $10 \%$, respectively and a master node always in listening mode (i.e. with full duty cycle equal to $100 \%$ ) have been taken into account. In this case, the transmitted phase values show a greater variation range, as it is pointed out in Figure 7, since the switching to the high power state from the low power state considerably effects the accurateness of the duty cycle. Nevertheless, the STAR MAC protocol is again able to limit its negative impact through a compensation performed by each node every synchronisation period. In Figure 7 it is possible to notice that the phase values for the joint synchronisation messages are always compensated and their summation is equal to a constant values, that is the phase value of the master node affected by a unnoticeable drift. This property is better explained in Figure 8 where the statistics of the phase drifts for the master node and the node are depicted. In particular, it is possible to notice that the ordinary node is effected by a remarkable phase drift, whose mean value is about $500 \mathrm{~ms}$, whilst the master node mean phase drift is on the opposite equal to $-500 \mathrm{~ms}$, thus pointing out the time compensation provided by the STAR MAC protocol. These advantages is more evident by noticing that a complete phase rotation takes about $6000 \mathrm{sec}$ for an ordinary node (see Figure 7, while, basing on the above mentioned mean value, a phase rotation period would be equal to $300 \mathrm{sec}$ if this node was completely isolated, that is, without the time synchronisation provided by the master node).

Figure 5 Transmitted phase for a couple of similar nodes

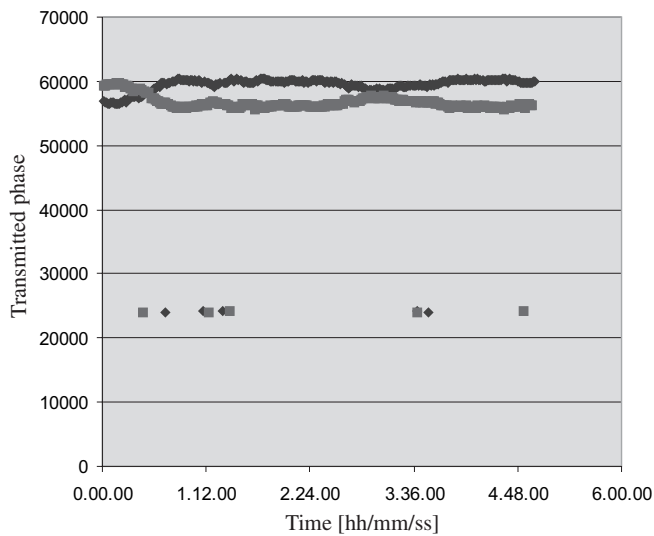


Figure 6 Statistics of the phase drift for similar nodes: (a) node 1 and (b) node 2

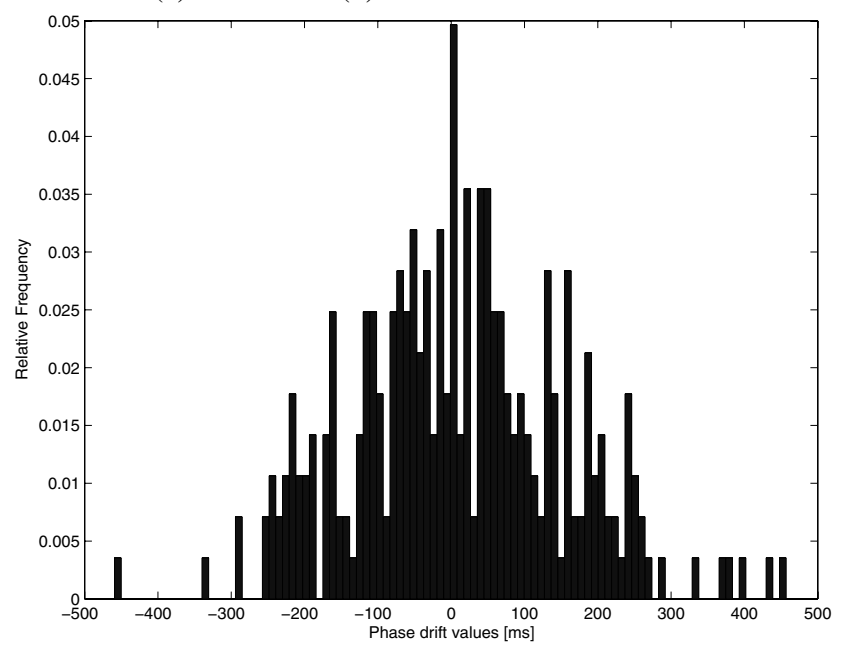

(a)

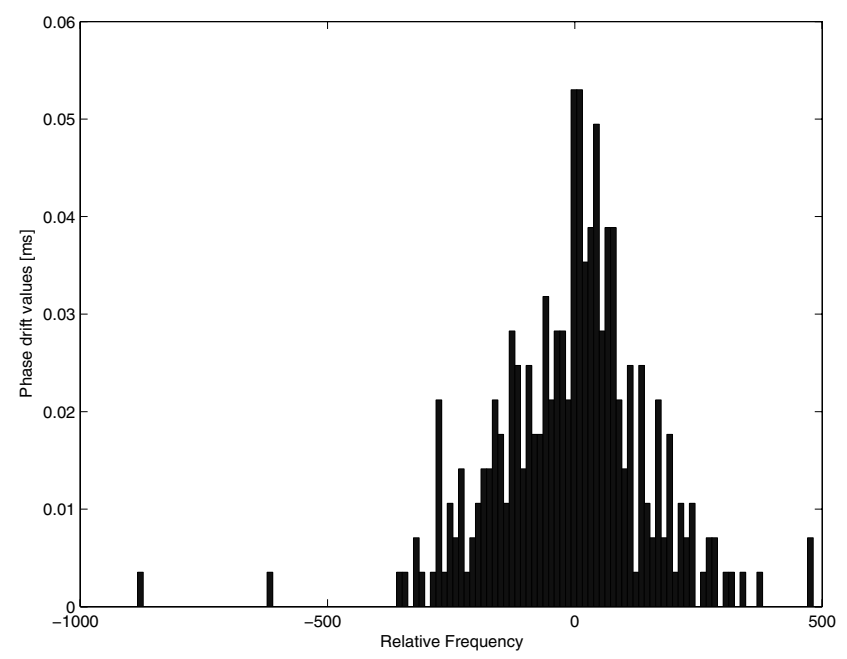

(b)

Figure 7 Transmitted phase for a couple of different nodes

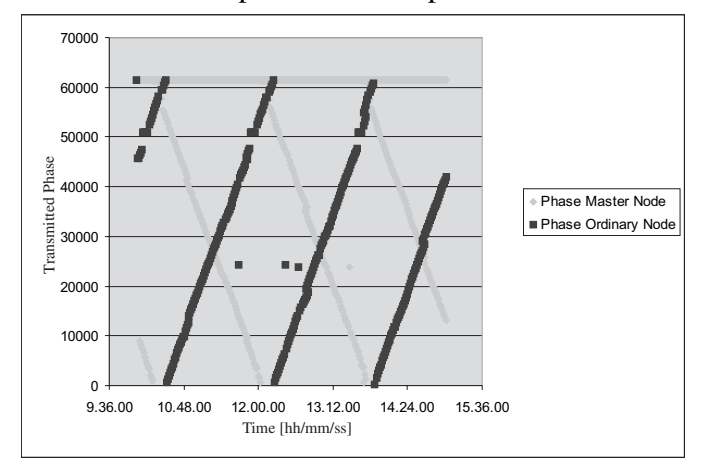

\subsection{STAR MAC protocol generalisation}

Generally speaking, the proposed STAR MAC scheme belongs to the class of distributed flat approaches, as each node might determine its own frame period and duty cycle, independently from its neighbours. This implies that the communication infrastructure is set up by means of progressively joining all the nodes two-by-two. Thus the network might be carried out in a modular way, without the need of a centralised authority coordinating all the nodes.
Moreover the system could better face several impairments throughout locally adapting the most relevant parameters (i.e. the listening and the sleeping time); in particular, they could be varied in a transparent way and the updated values might be delivered within one frame period. Finally, no strict hardware requirements in terms of clock accurateness are necessary.

Figure 8 Statistics of the phase drift for different nodes: (a) master node and (b) ordinary node

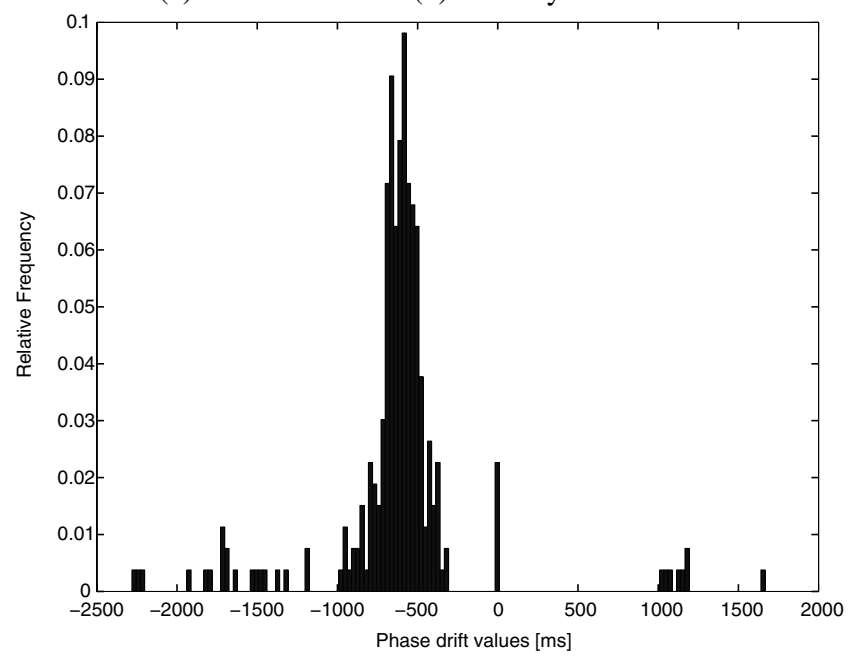

(a)

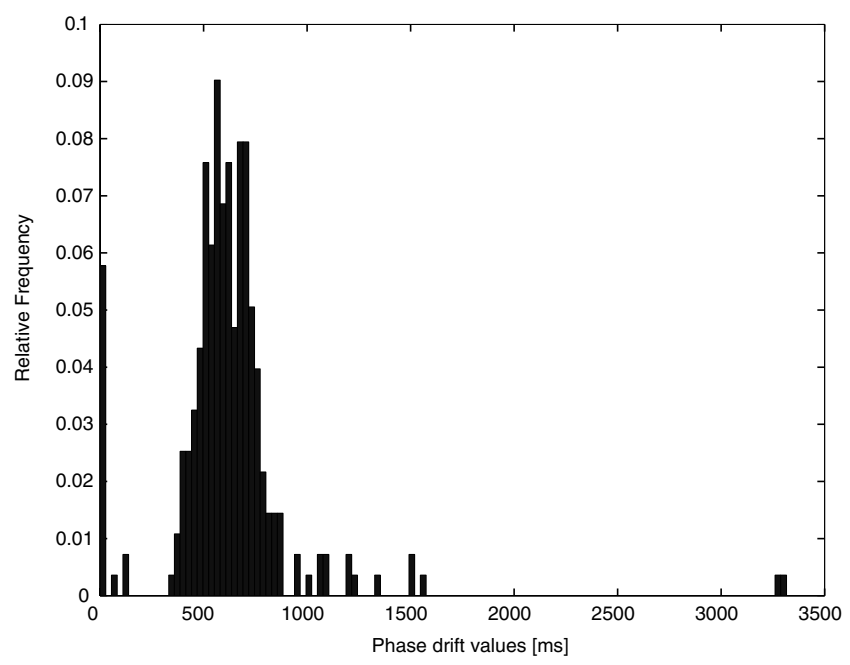

(b)

However, a deep investigation on the proposed STAR MAC robustness in the presence of different operative conditions need to be addressed. Specifically, two different test cases have been carried out within our system:

- Test 1: several nodes with different duty cycles, while the same $T_{\mathrm{f}}$, as described in Table 2 .

- $\quad$ Test 2: several nodes with different $T_{\mathrm{f}}$, while the same $T_{1}$, as summarised in Table 3.

With reference to the first scenario, the transmitted phase time diagram for several couple of nodes with different duty cycles have been derived for all the possible cases. In Figure 9, the master node $0(d=100 \%)$ and the ordinary node 22 $(d=1 \%)$ has been considered for what the phase drift analysis is concerned. It might be noticed that the phase 
drift is perfectly tracked, even if there is no perfect time alignment since the duty cycles are not the same. This advantage can be also pointed out in Figure 10, where the phase drift is reported for a couple of ordinary nodes, respectively, nodes 21 and 25 having quite different values of duty cycle, in particular $d_{21}=2 \%$ and $d_{25}=7 \%$.

Table 2 Parameters for Test 1

\begin{tabular}{lrrrr}
\hline$I D$ & $T_{l}$ & $T_{s}$ & $T_{f}$ & $d$ \\
\hline 0 & $100 \mathrm{sec}$ & $0 \mathrm{sec}$ & $100 \mathrm{sec}$ & $100 \%$ \\
21 & $2 \mathrm{sec}$ & $98 \mathrm{sec}$ & $100 \mathrm{sec}$ & $2 \%$ \\
22 & $1 \mathrm{sec}$ & $99 \mathrm{sec}$ & $100 \mathrm{sec}$ & $1 \%$ \\
23 & $3 \mathrm{sec}$ & $97 \mathrm{sec}$ & $100 \mathrm{sec}$ & $3 \%$ \\
24 & $5 \mathrm{sec}$ & $95 \mathrm{sec}$ & $100 \mathrm{sec}$ & $5 \%$ \\
25 & $7 \mathrm{sec}$ & $93 \mathrm{sec}$ & $100 \mathrm{sec}$ & $7 \%$ \\
\hline
\end{tabular}

Table 3 Parameters for Test 2

\begin{tabular}{llrrc}
\hline$I D$ & $T_{l}$ & $T_{s}$ & $T_{f}$ & $d$ \\
\hline 0 & $3 \mathrm{sec}$ & $107 \mathrm{sec}$ & $110 \mathrm{sec}$ & $100 \%$ \\
21 & $3 \mathrm{sec}$ & $33 \mathrm{sec}$ & $36 \mathrm{sec}$ & $8.3 \%$ \\
22 & $3 \mathrm{sec}$ & $47 \mathrm{sec}$ & $50 \mathrm{sec}$ & $6 \%$ \\
23 & $3 \mathrm{sec}$ & $59 \mathrm{sec}$ & $62 \mathrm{sec}$ & $4.8 \%$ \\
24 & $3 \mathrm{sec}$ & $71 \mathrm{sec}$ & $74 \mathrm{sec}$ & $4.05 \%$ \\
25 & $3 \mathrm{sec}$ & $99 \mathrm{sec}$ & $102 \mathrm{sec}$ & $2.9 \%$ \\
\hline
\end{tabular}

Figure 9 Transmitted phase in the case of different $d$ : (a) master node 0 and (b) ordinary node 22

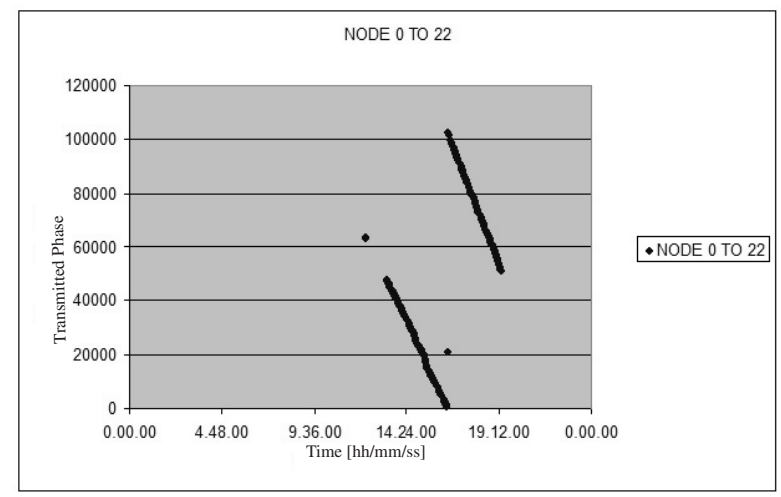

(a)

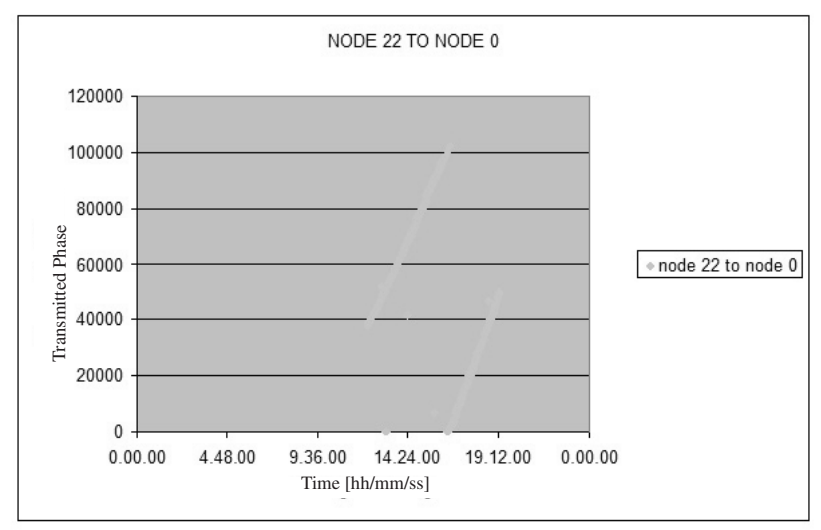

(b)

To complete the analysis of the STAR MAC protocol performance, the second test case has been taken into account, in which 6 nodes with independent $T_{\mathrm{f}}$ values are deployed. In this case, to the purpose of evaluating the phase drift compensation, it is not useful to refer to a time diagram as nodes have both different $d$ and $T_{\mathrm{f}}$ values, hence it is present a time shifting and a multienvelope effect due to several modulo- $T_{\mathrm{f}}$ operations. As a consequence, the time diagram is not easy to be interpreted.

Figure 10 Transmitted phase in the case of different $d$ : (a) master node 21 and (b) ordinary node 25

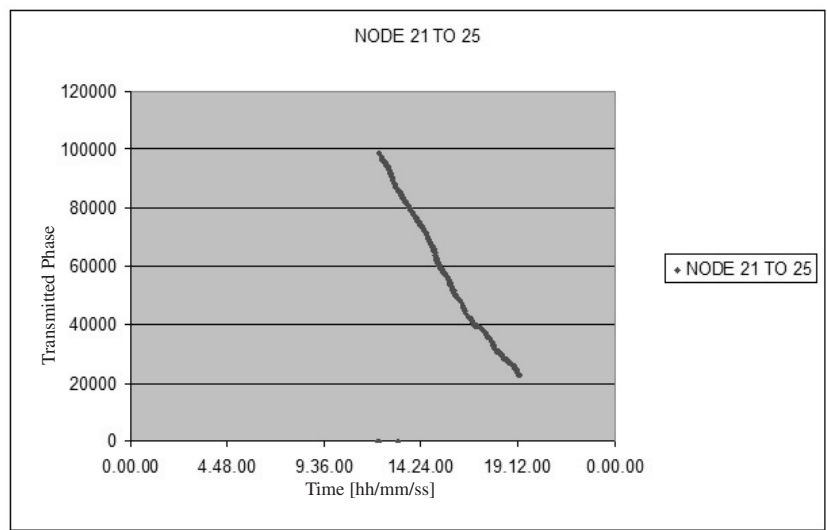

(a)

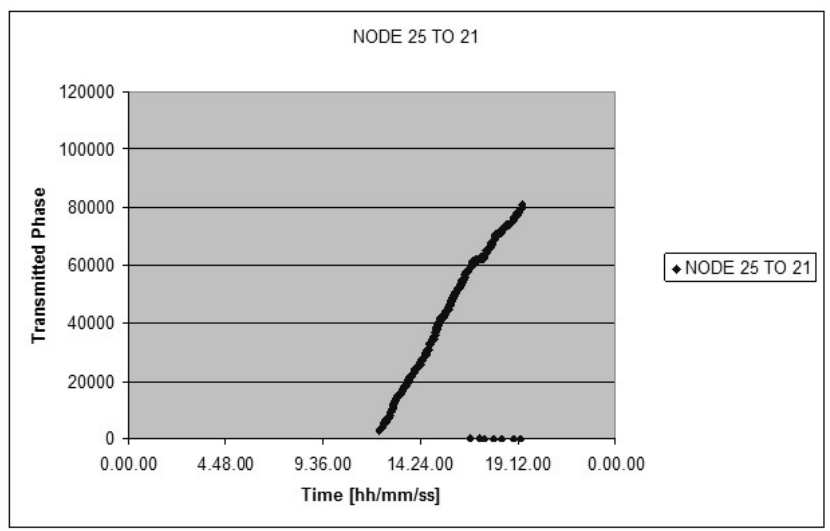

(b)

Otherwise, the STAR MAC connectivity maintenance might be better evaluated by considering the neighbours table size statistics as far as each node is concerned. In particular, in Figure 11, the probability density function (pdf) of having a certain number of neighbour nodes within the MAC table is shown. It is worth noticing that a generic node never gets disconnected and that the average table size is almost close to four elements, thus confirming the goodness of the proposed approach to increase the network connectivity, while reducing energy consumption without remarkable overhead increasing.

\section{Network layer protocol}

\subsection{Application requirements}

The provisioning of remote environmental monitoring capabilities, as expected within the GoodFood Project, establishes several requirements that communications protocols may satisfy. Some of them are related with the end-user applications characteristics, while the others 
depend on the node's hardware restrictions. First of all, the data reporting method is essentially time-driven Al-Karaki and Kamal (2004) as applications require periodic data monitoring. In future system releases it will be likely to support also query-driven scheme in which the gateway or another node in the network is able to generate a specific query to another node, to acquire a certain parameter value.

Figure 11 STAR MAC table size statistics

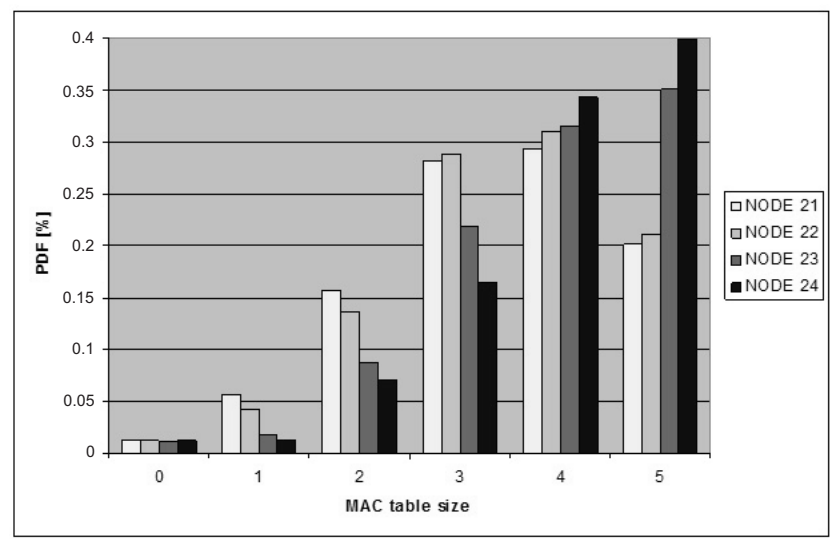

An additional requirement is represented by the need of fault tolerance, since a node may fail for several reasons. In this case, MAC and routing protocols might cooperate to quickly set up a new end-to-end path.

Besides a certain amount of redundancy might be introduced to allow data delivery even in the case of node's trouble due to physical damage or environmental interference. This is due to the fact that the communication protocol is unreliable, meaning that no explicit acknowledgement is provided in order to lower the signalling overhead and to preserve the available bandwidth. This affects the network layer performance rather than MAC layer.

Finally, scalability issues may be addressed to make the routing scheme able to operate in the correct way despite the number of deployed nodes that can be on the order of hundreds to sense a wide area. Moreover, the routing protocol might cope with contemporaneous transmissions, occurring when several nodes jointly enter the listening state trying to control the network congestion.

To end with, the power consumption is to be deeply considered in designing routing protocol, as usually nodes have limited energy supply and limited computing power. In a multihop WSN, each node plays a dual role as data sender and data router: the energy needed to perform computations and delivering information might dramatically reduce the node's lifetime, thus impacting on the network lifetime. As a results, energy-conserving forms of communication and computation are of primary importance.

\subsection{Proposed solutions}

In order to evaluate the capability of the proposed MAC scheme in establishing effective end-to-end communications within a WSN, a routing protocol has been introduced and integrated according to the cross-layer design principle (Shakkottai et al., 2003), where, in contrast to a strictly layered architecture, software layers interact more closely by means of information sharing. In particular, we refer to a proactive algorithm belonging to the class of link-state protocol that enhance the capabilities of the Link Estimation Parent Selection (LEPS) protocol (TinyOS, XXXX). It is based on periodically sending a control message to neighbour nodes to carry on information needed for building and maintaining the local routing table, depicted in Figure 12. In particular, it could be noticed the presence of several possible destinations (e.g. Sink ${ }_{1}$ and Sink $_{2}$ ), for each of them possible next hop candidates with the related parameters are listed. However, our approach resorts both to the signalling introduced by the MAC layer, that is, the synchronisation message and by the network layer, that is, the ping message, with the aim of minimising the overhead and make the system more adaptive in a cross-layer fashion. In particular, the parameters transmitted along a MAC synchronisation message, with period $T_{\mathrm{f}}$, are the following:

- next hop to reach the the gateway, that is, the MAC address of the one hop neighbour

- distance to the gateway in terms of number of needed hops

- phase the schedule time at which the neighbour become listening evaluated according to (2) or (3) in order to minimise the time latency

- link quality estimation as the ratio of correctly received and the expected synchronisation messages from a certain neighbour.

Figure 12 Routing table general structure

\begin{tabular}{|c|c|c|c|c|c|c|c|}
\hline Target & $\begin{array}{c}\text { Next } \\
\text { hop }\end{array}$ & $\begin{array}{c}\text { Hop } \\
\text { counter }\end{array}$ & Phase & Azimuth & $\begin{array}{c}\text { Link } \\
\text { quality }\end{array}$ & $\begin{array}{c}\text { Battery } \\
\text { level }\end{array}$ & $\begin{array}{c}\text { Congestion } \\
\text { level }\end{array}$ \\
\hline \multirow{2}{*}{ Sink $_{1}$} & $\mathrm{~A}$ & $N_{A}$ & $\varphi_{A}$ & $\alpha_{A}$ & $\eta_{A}$ & $B_{A}$ & $C_{A}$ \\
\cline { 2 - 8 } & $\mathrm{B}$ & $N_{B}$ & $\varphi_{B}$ & $\alpha_{B}$ & $\eta_{B}$ & $B_{B}$ & $C_{B}$ \\
\hline \multirow{2}{*}{ Sink $_{2}$} & $\mathrm{C}$ & $N_{C}$ & $\varphi_{C}$ & $\alpha_{C}$ & $\eta_{C}$ & $B_{C}$ & $C_{C}$ \\
\cline { 2 - 8 } & $\mathrm{D}$ & $N_{D}$ & $\varphi_{D}$ & $\alpha_{D}$ & $\eta_{D}$ & $B_{D}$ & $C_{D}$ \\
\hline
\end{tabular}

On the other hand, the parameters related to long-term phenomena are carried out by the Network layer messages (ping), with period $T_{\mathrm{p}} \gg T_{\mathrm{f}}$, in order to avoid unnecessary control traffics and thus, reducing congestion. Particularly, they are:

- battery level, that is, an estimation of the energy available at that node

- congestion level in terms of the ratio between the number of packets present in the local buffer and the maximum number of packets to be store in.

Once, the routing table has been filled with these parameters, it is possible to derive the proper metric by means of a weighted summation of them.

\subsection{Overall performance analysis}

\subsubsection{Test-case scenario}

The reference scenario considered in performing the simulations is related to the vineyard area selected for the exploitation of the GoodFood project Pilot Side at the 
University of Florence's owned Montepaldi farm. In this site, 15 nodes will be deployed in $10,000 \mathrm{~m}^{2}$ area by the end of 2005 and at the present 9 nodes are fully operative. Each node could be endowed with sensors monitoring three different environments: plant, root soil and canopy atmosphere. In Table 4, the characteristics of each sensed environment in terms of involved sensors and acquisition period are presented. On the whole nodes, the sensors for soil temperature and moisture, leaf temperature and air temperature/humidity will be implemented.

Table 4 Monitored parameters in the vineyard

\begin{tabular}{llc}
\hline Subsystem & \multicolumn{1}{c}{ Parameters } & $T_{Q}[\mathrm{~min}]$ \\
\hline \multirow{2}{*}{ Root soil } & Soil temperature & 15 \\
& Soil moisture & 15 \\
Canopy atmosphere & Air temperature & 15 \\
& Air humidity & 15 \\
Plant & Leaf temperature & 15 \\
& Shoot diametric growth & 15 \\
\hline
\end{tabular}

The power consumption values for the adopted optimised platform are those reported in Table 1; besides, $T_{1}=4 \mathrm{sec}$, $T_{\mathrm{S}}=56 \mathrm{sec}$ and consequently, $d=6.7 \%$, moreover, the deployed batteries are C-type primary lithium batteries with $7 \mathrm{Ah} 3 \mathrm{~V}$. Finally, the applied communications protocol is described in Section 3.2.

The whole system is still running unattended beginning from the summer season; packets are collected by the master node and their correct delivering is monitored by a specific application hosted within the remote server (logger) that stores and processes data.

\subsubsection{Experimental results}

First of all, has been measured the node's energetic consumption by averaging it over the whole network: it results to be equal to $1.44 \mathrm{mAh}$. On the other hand, its estimated value, based on modelling the network behaviour as a FSM and considering the practical power consumption values as in Table 1, is equal to $1.15 \mathrm{mAh}$, pointing out a good agreement with the experimental results. As a consequence, the node life time is greater than Seven months in the case of above mentioned battery adoption, that allows the network to operate for a farming season without maintenance, as it is claimed by the user requirements.

Then the Packet Delivery Ratio (PDR), that is, the ratio between the number of data packets correctly received by the master node and the number of sent packets estimated according to the data sampling rate, has been verified to be equal to 0.948 . It could be due to link failures or the residual packet collisions rate, not correctly handled by the STAR MAC protocol, as packets are retransmitted when the receiver is the sleeping mode. This allow the WSN utilisation for accurate environmental monitoring and implicitly, validate the efficiency of the adopted communication protocol.

To the purpose of evaluate the Quality of Service (QoS) of the cross-layer solution, it has been measured the average number of hops needed to deliver a packet to its destination. It has been made feasible by concatenating the identifier of intermediate nodes in a proper field of the packet header. It is equal to 2.83; this circumstance implies that almost all the data packets are not subject to loops that involve at least four hops and the routing functionalities are preserved. Finally, the measured mean delivery delay, that is the difference between the packet delivering time and the instant of its first sending, results to be equal to $60.1 \mathrm{sec}$, as the phase is randomly selected with uniform distribution and the queue waiting time might be neglected for a low loaded condition. This value represents a reasonable latency for the considered application in which slow varying phenomena are monitored.

\section{Conclusion}

This paper deals with the proposal of an efficient cross-layer communication protocol, allowing remarkable performance, in terms of low power consumption and QoS, for a WSN operating in a critical scenario like outdoor environmental monitoring. At this aim an energy efficient MAC protocol, namely STAR MAC, has been proposed and properly integrated within a routing scheme, minimising the signalling overhead. The protocol has been analytically characterised and generalised to the case of completely independent nodes in terms of frame period and duty cycle values. Further, it is modified in order to cope with densely deployed networks, giving rise to the Enhanced STAR MAC (STAR+). The performance of the overall multihop communication solution has been evaluated, by implementing it on motes operating in the operative scenario related to the EU Integrated Project GoodFood. In particular, it has been also described a practical implementation of the Pilot Site, in terms of adopted platforms and architectures on which the testing procedure has been performed. The experimental results highlighted a noticeable performance as far as energetic consumption, the node life time, the data delivering efficiency and latency are concerned. This allows the application of the WSN under investigation to the field of environmental monitoring.

\section{Acknowledgements}

The authors would like to express their gratitude to Professor Gianfranco Manes for his fruitful comments and discussions. A particular thank the members of the EU Integrated Project FP6-IST-1-508774-IP 'GoodFood' WorkPackage 7 for their supporting in this research.

\section{References}

Al-Karaki, J.N. and Kamal, A.E. (2004) 'Routing techniques in wireless sensor networks: a survey', IEEE Communication on Mag, December, No. 6, pp.6-28.

Akyildiz, I.F., Su, W., Sankarasubramaniam, Y. and Cayirci, E. (2002) 'A survey on sensor networks', IEEE Communication on Mag, August, Vol. 40, No. 8, pp.102-114. 
CC1150 (2006) Available at: http://www.chipcon.com.

El-Hoiydi, A., Decotignie, J-D., Enz, C. and Le Roux, E. (2003) 'WiseMAC, an ultra low power MAC protocol for the WiseNET wireless sensor network', Proceedings of Sensor System'03, June.

Hać, A. (2003) Wireless Sensor Networks Designs, John Wiley \& Sons.

IEEE standard 802.11 (1999) 'Wireless LAN medium access control (MAC) and physical layer (PHY) specifications'.

Langendoen, K. and Halkes, G. (2005) 'Energy-efficient medium access control', Technical Report, Delft University of Technology.

Lu, G., Krishnamachari, B. and Raghavendra, C. (2004) 'An adaptive energy-efficient and low-latency MAC for data gathering in sensor networks', Proceedings of WMAN'04, April.

Mattoli, V., Mondini, A., Razeeb, K.M., O'Flynn, B., Murphy, F., Bellis, S., Collodi, G., Manes, A., Pennacchia, P., Mazzolai, B. and Dario, P. (2005) 'Development of a programmable sensor interface for wireless network nodes for intelligent agricultural applications', Proceedings of IE'05, June.
MICA2 Series (XXXX) Available at: http://www.xbow.com/ Products.

Shakkottai, S., Rappaport, T.S. and Karlsson, P.C. (2003) 'Cross-layer design for wireless networks', IEEE Communication on Mag, October, Vol. 41, No. 10, pp.74-80.

TinyOS (XXXX) Available at: http://www.tinyos.net.

van Dam, T. and Langendoen, K. (2003) 'An adaptive energy-efficient MAC protocol for wireless sensor networks', Proceedings of Sensor System'03, November.

Ye, W., Heidemann, J. and Estrin, D. (2002) 'An energy-efficient MAC protocol for wireless sensor networks', Proceedings of INFOCOM'02, June.

\section{Note}

${ }^{1}$ As electromagnetic power can be measured in V A, it follows that energy might be expressed in VAs. Thus, once a certain voltage has been fixed (depending on the selected battery) energy can be characterised without ambiguity. 


\section{Appendix}

In order to point out the multicast gain provided by the proposed STAR+ approach, we derive the average number of subset $K$, that is, the average number of synchronisation messages delivered. We suppose that there are $N$ nodes and the average number of nodes belonging to a subset is $\bar{n}$. As a consequence, $K$ might be expressed as:

$$
K=\left\lceil\frac{N}{\bar{n}}\right\rceil
$$

To evaluate $\bar{n}$ we refer to Figure A1: in particular a couple of nodes, namely $i$ and $j$, belongs to the same subset if:

$$
\left|t_{i}-t_{j}\right| \leq T_{1}-T_{\mathrm{Rx}}
$$

where $t_{i}$ and $t_{j}$ are, respectively, the instants at which nodes $i$ and $j$ go into the listening state, $T_{1}$ is the listening period and $T_{\mathrm{Rx}}$ is the minimum time needed to receive a synchronisation message. For the sake of generality, we suppose that $t_{i}$ and $t_{j}$ are both random variable uniformly distributed over the interval $\left(0, T_{\mathrm{f}}\right]$. As a result, the probability that two nodes belong to the same subset is:

$$
P_{2}=\frac{2}{T_{\mathrm{f}}}\left(T_{1}-T_{\mathrm{Rx}}\right)
$$

Generalising (10) for the case of $n$ neighbours belonging to the same subset, we have:

$$
P_{n}=\left[\frac{2}{T_{\mathrm{f}}}\left(T_{1}-T_{\mathrm{Rx}}\right)\right]^{n-1}
$$

since the time each node enters the listening state is a random variables. Then the average number of nodes belonging to a generic subset can be expressed as:

$$
\bar{n}=\sum_{n=2}^{N-1} n\left[\frac{2}{T_{\mathrm{f}}}\left(T_{1}-T_{\mathrm{Rx}}\right)\right]^{n-1}
$$

By defining $q \doteq \frac{2}{T_{\mathrm{f}}}\left(T_{1}-T_{\mathrm{Rx}}\right)$, it yields:

$$
\bar{n}=\frac{(N-1) q^{N}-N q^{N-1}+2 q^{3}-q^{2}-4 q+2}{(1-q)^{2}}
$$

The derivation of $K$ is straightforward by substituting (12) into (8).

Figure A1: $\quad$ Subset derivation procedure for nodes $i$ and $j$

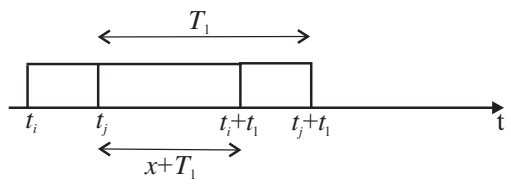

